\title{
Dynamic and biocompatible
} thermo-responsive magnetic

\section{hydrogels that respond to an alternating magnetic field}

\section{Journal Article}

\section{Author(s):}

Crippa, Federica; Moore, Thomas L.; Mortato, Mariangela; Geers, Christoph; Haeni, Laetitia; Hirt, Ann Marie (D); RothenRutishauser, Barbara; Petri-Fink, Alke

Publication date:

2017-04-01

Permanent link:

https://doi.org/10.3929/ethz-b-000218493

Rights / license:

Creative Commons Attribution-NonCommercial-NoDerivatives 4.0 International

Originally published in:

Journal of Magnetism and Magnetic Materials 427, https://doi.org/10.1016/j.jmmm.2016.11.023 


\title{
Dynamic and biocompatible thermo-responsive magnetic hydrogels that respond to an alternating magnetic field.
}

Federica Crippa $^{\mathrm{a}}$, Thomas L. Moore ${ }^{\mathrm{a}}$, Mariangela Mortato ${ }^{\mathrm{a}}$, Christoph Geers ${ }^{\mathrm{a}}$, Laetitia Haeni ${ }^{\mathrm{a}}$, Ann M. Hirt $^{\mathrm{b}}$, Barbara Rothen-Rutishauser ${ }^{\mathrm{a}}$, Alke Petri-Fink ${ }^{\mathrm{a}, \mathrm{c}, *}$

${ }^{a}$ Adolphe Merkle Institute, University of Fribourg, Chemin des Verdiers 4, CH-1700 Fribourg, Switzerland

${ }^{b}$ Institute for Geophysics, ETH Zurich, Sonneggstrasse 5, CH-8092 Zurich, Switzerland

${ }^{c}$ Chemistry Department, University of Fribourg, Chemin du Musée 9, CH-1700 Fribourg Switzerland

* Corresponding author: Chemistry Department, University of Fribourg, Chemin du Musée 9, CH-1700 Fribourg, Switzerland. E-mail: alke.fink@unifr.ch (A. Petri-Fink).

Keywords: Magnetic hydrogels, magnetic nanoparticles, hyperthermia, stimuli-responsive substrates, mechanobiology.

\begin{abstract}
Magnetic thermo-responsive hydrogels are a new class of materials that have recently attracted interest in biomedicine due to their ability to change phase upon magnetic stimulation. They have been used for drug release, magnetic hyperthermia treatment, and can potentially be engineered as stimuli-responsive substrates for cell mechanobiology. In this regard, we propose a series of magnetic thermo-responsive nanocomposite substrates that undergo cyclical swelling and de-swelling phases when actuated by an alternating magnetic field in aqueous environment. The synthetized substrates are obtained with a facile and reproducible method from poly-N-isopropylacrylamide and superparamagnetic iron oxide nanoparticles. Their conformation and the temperature-related, magnetic, and biological behaviors were characterized via scanning electron microscopy, swelling ratio analysis, vibrating sample magnetometry, alternating magnetic field stimulation and indirect viability assays. The nanocomposites showed no cytotoxicity with fibroblast cells, and exhibited swelling/de-swelling behavior near physiological temperatures (around $34{ }^{\circ} \mathrm{C}$ ). Therefore these magnetic thermo-responsive hydrogels are promising materials as stimuli responsive substrates allowing the study of cell-behavior by changing the hydrogel properties in situ.
\end{abstract}

\section{Introduction}

Hydrogels, hydrophilic polymer networks which can absorb $>10-30 \%$ of their dry weight in water, are attracting considerable interest in biomedicine and as biomaterials due to their intrinsic properties such as 
biocompatibility, structure, and chemical and mechanical behavior [1]-[4]. Moreover, these materials can be tailored to possess properties similar to that of native biological tissues. Recently a novel class of hydrogels, which offer additional features, has been proposed: magnetic hydrogels that are composite materials comprised of magnetic nano- or microparticles incorporated within a polymeric matrix[5]. In biomedicine, these magnetic hydrogels have been investigated for a variety of applications. For example, magnetic actuators that combine high elasticity and magnetic behavior have been developed to mimic skeletal muscles behavior[6], and magnetic bio-hybrid scaffolds with improved physico-chemical and mechanical properties have been engineered for bone regeneration[7]. Furthermore an emerging application of magnetic hydrogels is to implement superparamagnetic iron oxide nanoparticles (SPIONs) as a functional, stimuli-responsive component within thermo-responsive hydrogels[8], [9]. Thermoresponsive hydrogels are materials that swell or collapse upon temperature changes, and by incorporating SPIONs it is possible to magnetically trigger this transition.

SPIONs with a core diameter between 5 to $28 \mathrm{~nm}$ [10], when exposed to an alternating magnetic field (AMF), dissipate heat though Néel and Brownian relaxation phenomena[11], [12]. When incorporated into a thermo-responsive hydrogel, the heat is then transferred to the surrounding polymeric matrix thereby inducing conformational change and polymer collapse[13]. These magnetic hydrogels have a reversible behavior and therefore can re-acquire the initial conformation when the AMF is off [14]. Several groups have thus developed remotely triggered drug delivery systems with micro-gels [15], [16], injectable hydrogels [17] and solid patches that can release drug when exposed to an AMF [14], [18]. Others have proposed hydrogels that potentially can target and heat a tumor for hyperthermia treatment [19].

However hyperthermia treatments and drug delivery technologies are not the only fields that benefit from magneto thermo-hydrogels. In tissue engineering, and specifically mechanobiology, the temporal variations of a substrate surface are thought to be fundamental in governing cell adhesion, morphology and differentiation[20]-[22]. Hence to mimic the natural cells' environment that undergoes remodeling in physiological or pathological conditions [23], [24], a classical petri dish or a static cell substrate with defined mechanical properties is not sufficient. To overcome this limitation and study the cellular reaction to mechanical environmental changes, several dynamic cell-culture substrates, in which stiffness and topography can be tuned in situ, have been developed [20], [25], [26].

In the growing field of dynamic, stimuli-responsive cell substrates there are only a few which involve the use of magnetic hydrogels [27]-[29] and none rely on AMF stimulation. In particular, acrylamide gels loaded with nickel micro-wires were shown to alter their surface roughness upon the application of a static magnetic field and to induce changes in the adhesion area of vascular smooth cells [27]. Another study [29] focused on the development of a magneto-active elastomer that changes stiffness 
and topography after application of static and oscillating field. This substrate was used to study migration and morphology of human fibroblasts. Moreover, cylindrical micro-pillars made of iron oxide nanoparticles-loaded poly(caprolactone)-based polymer substrates were bent and extended with the use of an AMF. However in the presence of cells, the morphology of the substrate was triggered only by a direct change in the temperature of the cell culture medium and not by the AMF [28]. Thus, by including SPIONs directly into a thermo-responsive hydrogel it is possible to trigger mechanical changes in a hydrogel substrate via an AMF.

Here we report a magnetic, thermo-responsive model substrate based on poly-Nisopropylacrylamide (poly-NIPAM) embedded with SPIONs that reversibly changes phase upon AMF stimulation. The hydrogels were prepared as films covalently crosslinked to a glass coverslip, each containing different concentrations of nanoparticles. These materials combine good thermal behavior (change swelling and de-swelling behavior over a narrow temperature range), superparamagnetic properties, and have a high degree of biocompatibility. When excited with AMF they show a de-swelling/ swelling reversible process at physiological temperatures. Moreover, variations of the substrate mechanical properties (e.g. hydrogel elasticity and topography) can be related to the hydrogel phase change [25], [30]. The hydrogels were biocompatible in both the swollen and de-swollen states as shown by a biocompatibility test for murine fibroblasts in vitro. Hence the proposed materials have the potential to be used as stimuli-responsive substrates for cell culture.

\section{Material and methods}

\subsection{Materials}

\subsubsection{Chemicals}

All chemicals were of analytical reagent grade unless further specified and were used without any purification except for the monomer N-isopropylacrylamide (NIPAM) which was purified prior to use. For NIPAM purification, $20 \mathrm{~g}$ was pre-purified by recrystallization with hexane $(300 \mathrm{ml})$. Then the precipitate was filtered and washed with ice-cold hexane to give colorless crystals (19 g); the final monomer was recollected and stored at $4^{\circ} \mathrm{C}$. Iron(III) chloride hexahydrate (99\%), oleic acid (technical grade, 90\%), citric acid (99.5\%), N,N-dimethylformamide (DMF, 99.8\%), 1,2-dichlorobenzene (DCB, 99\%), N,N’-Methylenebis(acrylamide) (BIS, 99\%, Mw=154.17), ammonium persulfate (APS, 98\%) hydrochloric acid ( $\mathrm{HCl}, 37 \%)$, nitric acid $\left(\mathrm{HNO}_{3}, 65 \%\right)$, potassium hexacyanoferrate(II) trihydrate (98.5\%) and diethyl ether (99\%) were supplied by Sigma-Aldrich. Sodium oleate (97\%) and tri-noctylamine (97\%) were purchased from TCI Europe N.V. Absolute ethanol, acetone and 2-propanol were purchased from VWR Chemicals. 3-(trimethoxysilyl)propylmethacrylate (TPM) was purchased from 
ABCR GmbH and acetic acid (glacial, 100\%) from Merck. N,N,N,N'-tetramethylethylenediamine (TEMED) was obtained from Bio-Rad and N-Isopropylacrylamide (NIPAM, 99\%, Mw=113.16) from ACROS Organics. All aqueous solutions were prepared with deionized water obtained from a Milli-Q system (resistivity=18.2 $\Omega$, Millipore AG).

\subsubsection{Cell culture}

NIH-3T3 murine fibroblasts were obtained from American Type Culture Collection (ATCC). Cells were cultured in Dulbecco's Modified Eagle’s Medium supplemented with 10\% v/v calf bovine serum (ATCC ${ }^{\circledR} 30-2030^{\mathrm{TM}}$ ), 2mM L-glutamine and $1 \% \mathrm{v} / \mathrm{v}$ Penicillin-Streptomycin at $37{ }^{\circ} \mathrm{C}$ and $5 \% \mathrm{CO}_{2}$ in a humidified incubator.

\subsection{SPIONs synthesis}

SPIONs with a core diameter of $21.8 \pm 0.9 \mathrm{~nm}$ (average \pm SD, $n=1258$ ) were synthetized by thermal decomposition according to a modified procedure reported by Park, et al [31]. In brief, an iron oleate complex was prepared reacting iron chloride $\left(\mathrm{FeCl}_{3} \cdot 6 \mathrm{H}_{2} \mathrm{O}\right)$ with sodium oleate. Then the complex was thermally decomposed in presence of oleic acid at $320^{\circ} \mathrm{C}$ in tri-n-octylamine for 1 hour (details in Supplementary Information). The solution was rapidly cooled down, and the nanoparticles were separated and washed by sequential centrifugations. Resulting oleic acid coated nanoparticles were re-dispersed in hexane and stored at $4{ }^{\circ} \mathrm{C}$.

\subsection{SPIONs functionalization}

A ligand exchange procedure was performed in order to exchange oleic acid on the SPION surface with citric acid, thereby yielding nanoparticles colloidally stable in polar (i.e. aqueous) solvents and suitable for incorporation in the polymer [32]. For this, the SPIONs were dispersed in a solution of DCB and DMF (ratio 1:1). Citric acid was then added (0.8 mg citric acid/mg Fe) and the solution was stirred for 24 hours at $100^{\circ} \mathrm{C}$. Following this, the nanoparticles were precipitated in $200 \mathrm{~mL}$ of diethyl ether and recovered with a magnet (Nickel-plated NdFeB, Supermagnete). The resulting citric acid coated SPIONs were washed in acetone and finally re-dispersed in Milli-Q water.

\subsection{Transmission electron microscopy}

The size and morphology of citric acid SPIONs was investigated by transmission electron microscopy (TEM). Samples were prepared drying the nanoparticle suspensions on copper carbon-coated mesh grids. Micrographs were acquired with a Tecnai Spirit transmission electron microscope (FEI) operating at 120 
$\mathrm{kV}$ and equipped with a Veleta CCD camera (Olympus). The core diameters of the nanoparticles were estimated with automatized size distribution analysis software (ImageJ, National Institutes of Health).

\subsection{Dynamic light scattering}

SPIONs investigation of size and colloidal stability was performed with a dynamic light scattering setup (3D LS Spectrometer, LS Instruments AG). The suspension was diluted to a low concentration (0.05 $\mathrm{mg} / \mathrm{ml} \mathrm{Fe}$ ) to avoid multiple light scattering. Data were collected at $25^{\circ} \mathrm{C}$ with a scattering angle of $90^{\circ}$ and were analyzed with a customized script in Mathematica (Version 10.1, Wolfram Research Inc).

\subsection{Iron quantification (Prussian Blue colorimetric assay)}

The iron concentration of citric acid coated SPIONs was quantified using the Prussian Blue colorimetric assay [33]. Nanoparticles were dissolved in a $6 \mathrm{M} \mathrm{HCl}$ and $0.01 \mathrm{M} \mathrm{HNO}_{3}$ solution. Then, 5\% w/v of freshly prepared potassium hexacyanoferrate(II) trihydrate was added and the absorbance at $690 \mathrm{~nm}$ was read after 10 minutes on a VICTOR3, 1420 Multilabel Counter (Perkin Elmer). Each experiment was repeated in triplicate at least three times.

\subsection{Synthesis of PNIPAM-SPIONs hydrogels}

\subsubsection{Coverslips functionalization}

Glass treatment was necessary to guarantee the covalent attachment between the hydrogel and the coverslip and was adapted from a procedure by Vignaud et al [34]. Round glass coverslips (diameter $=22$ $\mathrm{mm}$ ) were cleaned and sonicated with acetone and 2-propanol for 30 minutes. A solution of $0.5 \%$ TPM in $100 \mathrm{ml}$ of ethanol was prepared. Then $3 \mathrm{ml}$ of acetic acid (1:10 in Milli-Q water) were added and the coverslips were immersed in the final solution for 5 minutes. After the coverslips were rinsed with absolute ethanol and dried with nitrogen.

\subsubsection{Hydrogels synthesis}

Poly-NIPAM hydrogels loaded with SPIONS at $0,1,2.5$ and 5\% w/w of SPIONs to the monomers NIPAM and BIS, were synthesized by radical polymerization. SPIONs, NIPAM and BIS (monomers), and TEMED (catalyst) were mixed in varying ratios (Table 1) in Milli-Q $\mathrm{H}_{2} \mathrm{O}$ and sonicated in a flask for 5 minutes under inert atmosphere. APS at $10 \% \mathrm{w} / \mathrm{v}$ was added as an initiator. To prepare a flat substrate suitable for cell culture, $250 \mu \mathrm{L}$ of the polymerization mix were sandwiched between two glass coverslips (one functionalized with TPM, the other not). Upon complete polymerization the non-functionalized coverslip was removed and the gel was washed in Milli-Q water for three days under gentle agitation. The final substrates were flat hydrogels (thickness $\approx 0.7 \mathrm{~mm}$, diameter $\approx 20 \mathrm{~mm}$ ) covalently attached on one 
side to a glass coverslip. To perform bulk measures on the hydrogels, the polymerization solutions were cast in cylindrical containers (height $\approx 20 \mathrm{~mm}$, diameter $\approx 8 \mathrm{~mm}$ ). At the end, the hydrogels were extracted, washed, cut in smaller pieces and freeze-dried.

Table 1 Summary of synthetic parameters.

\begin{tabular}{|c|c|c|c|c|c|c|}
\hline & NIPAM (mg) & BIS (mg) & TEMED $(\mu \mathrm{l})$ & $\begin{array}{c}\text { Milli-Q Water } \\
\text { (ml) }\end{array}$ & $\begin{array}{c}\text { SPIONs (ml) } \\
\text { conc. }=8.46 \mathrm{mg} / \mathrm{ml}\end{array}$ & $\begin{array}{c}\text { APS 10\% } \\
w / v(\mu l)\end{array}$ \\
\hline PNIPAM 0\% & 318 & 4.5 & 1.88 & 3 & 0 & 18.75 \\
\hline $\begin{array}{c}\text { PNIPAM- } \\
\text { SPIONs 1\% }\end{array}$ & 318 & 4.5 & 1.88 & 2.62 & 0.38 & 18.75 \\
\hline $\begin{array}{c}\text { PNIPAM- } \\
\text { SPIONs } 2.5 \%\end{array}$ & 318 & 4.5 & 1.88 & 2.05 & 0.95 & 18.75 \\
\hline $\begin{array}{c}\text { PNIPAM- } \\
\text { SPIONs 5\% }\end{array}$ & 318 & 4.5 & 1.88 & 1.1 & 1.9 & 18.75 \\
\hline
\end{tabular}

\subsection{De-swelling characterization}

Swollen samples at room temperature were allowed to de-swell in a thermostatic bath from room temperature to $55{ }^{\circ} \mathrm{C}\left(\Delta \mathrm{T}=5^{\circ} \mathrm{C}\right)$. Every temperature was kept constant for 24 hours and the sample weight $\left(\mathrm{W}_{\text {wet(T) }}\right)$ was analyzed gravimetrically [35]. After the test, the samples were freeze-dried and their dry weight was calculated $\left(\mathrm{W}_{\text {dry }}\right)$. The mass swelling ratio $(\mathrm{Q})$ as a function of the temperature was calculated using the following formula:

$\mathrm{Q}=\frac{\mathrm{W}_{\mathrm{wet}(\mathrm{T})}-\mathrm{W}_{\mathrm{dry}}}{\mathrm{W}_{\mathrm{dry}}}$.

\subsection{Vibrating sample magnetometry}

The magnetic properties of the citric acid SPIONs and the hydrogels were investigated with a vibrating sample magnetometer (VSM, Model 3900, Princeton Measurements Corporation). Citric acid SPIONs samples (iron mass $=42 \mu \mathrm{g}$ ) were dried on hydrophobic cotton pellets and fixed on the sample holder with Si-grease. Magnetic gels, in their hydrated state, were cast in plastic capsules and fixed to the sample holder with glue. The hysteresis loops were recorded using a measurement averaging time of $300 \mathrm{~nm}$ and a constant field increment. Magnetization was measured at room temperature as a function of the magnetic field. Data were collected and the magnetization values were normalized by the iron mass (for citric acid SPIONs) or by the dried sample mass (for the hydrogels). 


\subsection{Scanning electron microscopy (SEM)}

The hydrogels samples were freeze-dried, coated with a $4 \mathrm{~nm}$ layer of gold and investigated with a scanning electron microscope (Mira3 LM FE, Tescan). The images were obtained at 2.0k and 23.3k X magnification with an SE detector.

\subsection{Hyperthermia characterization}

All magnetic field experiments were performed on a commercial AMF generator (Magnetherm V1.5, Nanotherics Ltd) operating at $523.5 \mathrm{kHz}, 14.3 \mathrm{kA} / \mathrm{m}$ for the particles and at $110.0 \mathrm{kHz}, 19.9 \mathrm{kA} / \mathrm{m}$ or $523.0 \mathrm{kHz}, 15.9 \mathrm{kA} / \mathrm{m}$ for the gels. Citrate SPIONs were loaded in Styrofoam insulated sample holders and prior to every experiment they were let equilibrate at $19-20^{\circ} \mathrm{C}$. During the magnetic stimulation, changes in temperature were recorded for 60 seconds with fiber optic cables (device: FOB100, fiber optic sensors: FOBS-2, Omega Engineering). The heating slopes were extracted by interpolating the first 12 seconds of the signal with a linear fit and Specific Absorption Rate (SAR) and Intrinsic Loss Power (ILP) were calculated as previously described [36]. The hydrogels' temperature was recorded with fiber optic cables inserted in the samples (c.a. $0.2 \mathrm{~mm}$ under the surface of the hydrogel). The tests were carried on in

water at least in triplicates to ensure reproducibility. Two different sets of experiments were performed: (1) temperature increase upon magnetic stimulation was assessed with room temperature tests and (2) AMF-triggered phase change was obtained for the hydrogel containing 5\% of SPIONs after having stabilized it in water at temperatures around $33{ }^{\circ} \mathrm{C}$. For room temperature experiments the samples were allowed to equilibrate in Milli-Q water for 30 minutes and then tested with one or more magnetic cycles, defined as AMF on for 15 minutes and then off for 5 minutes. For phase change experiments the hydrogels were positioned inside a temperature-controlled sample holder designed to fit in the middle of the coil. In this case the gels were allowed to equilibrate for 1 hour and then excited with magnetic cycles (AMF on for 15 minutes and off for 10 minutes). Temperature was recorded (acquisition frequency $=0.6$ s) and pictures were taken every 5 minutes with a stereo microscope (AmScope) equipped with a digital camera (Model: FMA050, AmScope).

\subsection{Cell viability}

Cytotoxicity of potential leachables from the synthesized magnetic hydrogels was tested following ISO 10993-5 guidelines [37]. NIH 3T3 murine fibroblasts were seeded at 10,000 cells/well $\left(31,250 \mathrm{cells} / \mathrm{cm}^{2}\right)$ in a 96-well plate. For each tests three repeats were seeded per formulation and experiments were done in triplicate. PNIPAM-SPIONs 5\% were washed in sterile filtered $(0.2 \mu \mathrm{m})$ Milli-Q water and sterilized under UV light for 30 minutes. Gels of approximately $50 \mathrm{~mm}^{3}$ were immersed in $1 \mathrm{~mL}$ of complete cell 
culture media. Samples were immersed in media for 24 hours at $37^{\circ} \mathrm{C}$ or $25^{\circ} \mathrm{C}$ under constant stirring. To test whether swelling/de-swelling resulted in the leaching of toxic leachables, gels were incubated for 23 hours under constant stirring at $25^{\circ} \mathrm{C}$ and then subjected to three swelling/de-swelling cycles (15 min at $37^{\circ} \mathrm{C}$, and $5 \mathrm{~min}$ at $25^{\circ} \mathrm{C}$ ). As a positive cytotoxicity control, cells were exposed to $0.2 \mathrm{v} / \mathrm{v} \%$ Triton X100. For control experiments, 3T3 cells were exposed to varying concentrations of hydrogel pre-cursor monomers (NIPAM or BIS).

After treatment, $50 \mu \mathrm{L}$ of supernatant from each well was collected and viability was measured via a standard lactate dehydrogenase (LDH) cytotoxicity assay (Roche). A second viability assay was conducted based on the conversion of weakly fluorescent resazurin to highly fluorescent resorufin via active cellular respiration [38]. Cell culture media was removed and replaced with $100 \mu \mathrm{L}$ of resazurin dissolved at $88 \mu \mathrm{M}$ in complete cell culture media. Cells were incubated protected from light for 3 hours at $37{ }^{\circ} \mathrm{C}$ and $5 \% \mathrm{CO}_{2}$. Fluorescent signal was measured on a microplate reader (TriStar LB 941, Berthold Technologies) at $\lambda_{\mathrm{ex}}=560 \mathrm{~nm}$ and $\lambda_{\mathrm{em}}=590 \mathrm{~nm}$. For each formulation, samples were compared as a fraction (LDH assay) or percentage (resazurin assay) of the mean negative control, i.e. the average of the cells treated with only media. Statistical significance was determined via a one-way ANOVA comparison of means and Tukey's honestly significant difference test ( $p$-value $=0.05)$.

\section{Results and discussion}

\subsection{Nanoparticles synthesis and characterization}

SPIONs were synthesized by thermal decomposition of an iron oleate complex following a previously developed protocol [31]. After synthesis the nanoparticles were transferred to water through a ligand exchange procedure [32] that involved the replacement of surface grafted oleic acid with a citric acid. The obtained nanoparticles were dispersed in Milli-Q water and they formed a stable colloidal suspension. SPIONs' size and morphology were studied with TEM and DLS (Fig. 1A, B). The particles were spherical single nanocrystals with an average core diameter of about $22 \mathrm{~nm}$, suitable for magnetic hyperthermia [10] and a hydrodynamic diameter of $26 \mathrm{~nm}$ (polydispersity index = 24\%). Discrepancies in size distribution between TEM and DLS are typically observed for small nanoparticles and are related to the formation of a hydration shell around the nanoparticle surface, the electric double layer (a layer of ions and counter ions surrounding the particle due to the particle surface charge), and the presence of any coating molecules on the nanoparticle's surface [39]-[42]. However, with a citric acid coating it is not expected that these differences will be large (around 1-5 nm). Their magnetization was measured as a function of the magnetic field using VSM at room temperature (Fig. 1C). Particles showed a superparamagnetic behavior with not significant coercivity and had a saturation magnetization of $66.3 \pm 1.9 \mathrm{emu} / \mathrm{g}$ Fe (average $\pm \mathrm{SD}$, 
$\mathrm{n}=3$ ). The saturation magnetization value was lower than expected for bulk magnetite (92 emu/g) or for similar particles [43], [44]. Indeed it has been reported that nanoparticles synthesized by thermal decomposition can contain paramagnetic impurities in the magnetite crystal [45] and the lattice imperfection could determines a decrease in the saturation magnetization. SAR values of $139.3 \pm 3.59$ W/g Fe (average $\pm \mathrm{SD}, \mathrm{n}=3$ ) were obtained upon AMF stimulation ( $\mathrm{f}=523.5 \mathrm{kHz}, \mathrm{H}=14.3 \mathrm{kA} / \mathrm{m}$ ) and corresponded to an ILP of $1.3 \pm 0.03 \mathrm{nHm}^{2} / \mathrm{kg}$ Fe.
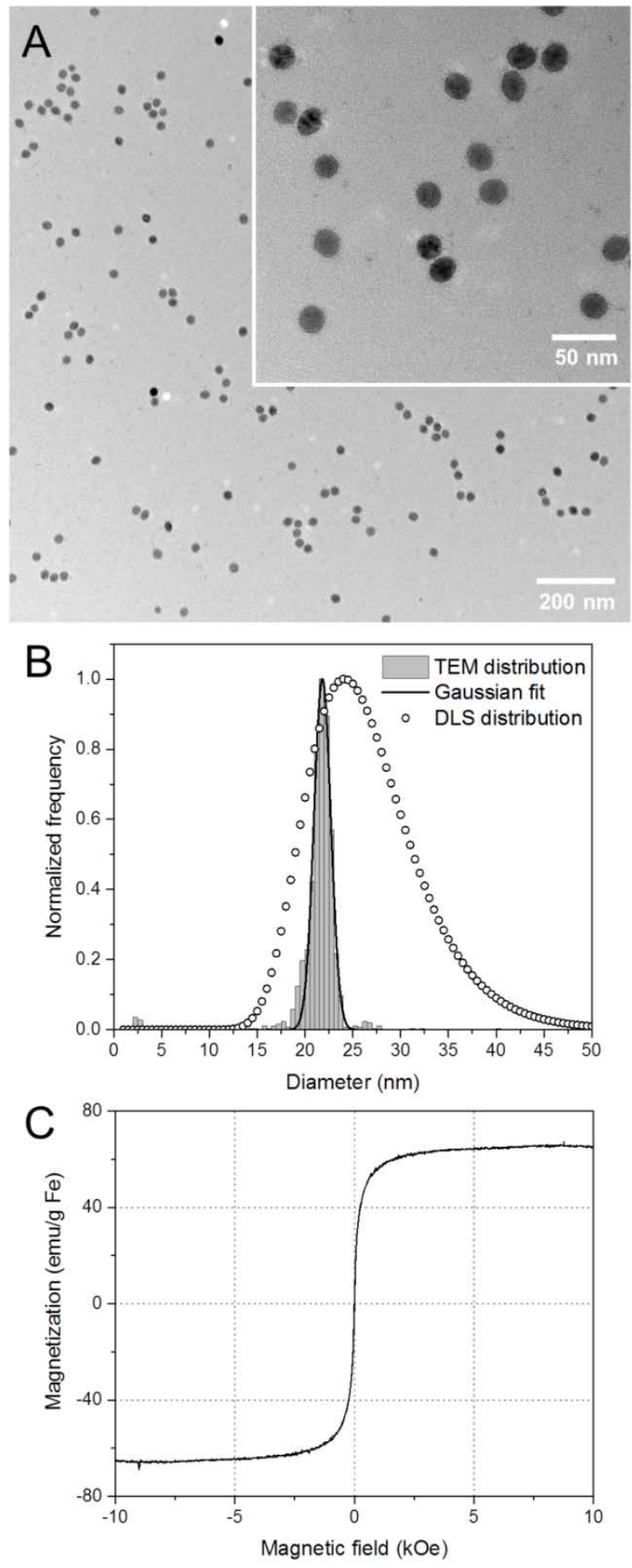
Fig. 1. TEM micrographs (A) illustrating the morphology of citric acid coated SPIONs. Particle size distributions obtained by TEM (correlated with the Gaussian fit) and by DLS (B). Magnetization MH curve of the citric acid coated SPIONs (C).

\subsection{Magnetic hydrogels characterization}

After synthesis all the magnetic hydrogels were characterized for morphology and thermal and magnetic properties. The SPIONs' distribution in the hydrogels was investigated with scanning electron microscopy (SEM) (Fig. 2). Particles were visible and showed an increase in number for PNIPAM loaded with 1, 2.5 and $5 \% \mathrm{w} / \mathrm{w}$ of SPIONs. Although the images were obtained close to the instrument detection limit (5 $\mathrm{nm}$ ), single SPIONs could be well detected. Therefore from the images it was possible to conclude that the particles were fairly evenly distributed and not agglomerated in the polymeric matrix even for the highest concentration (PNIPAM-SPIONs 5\%).
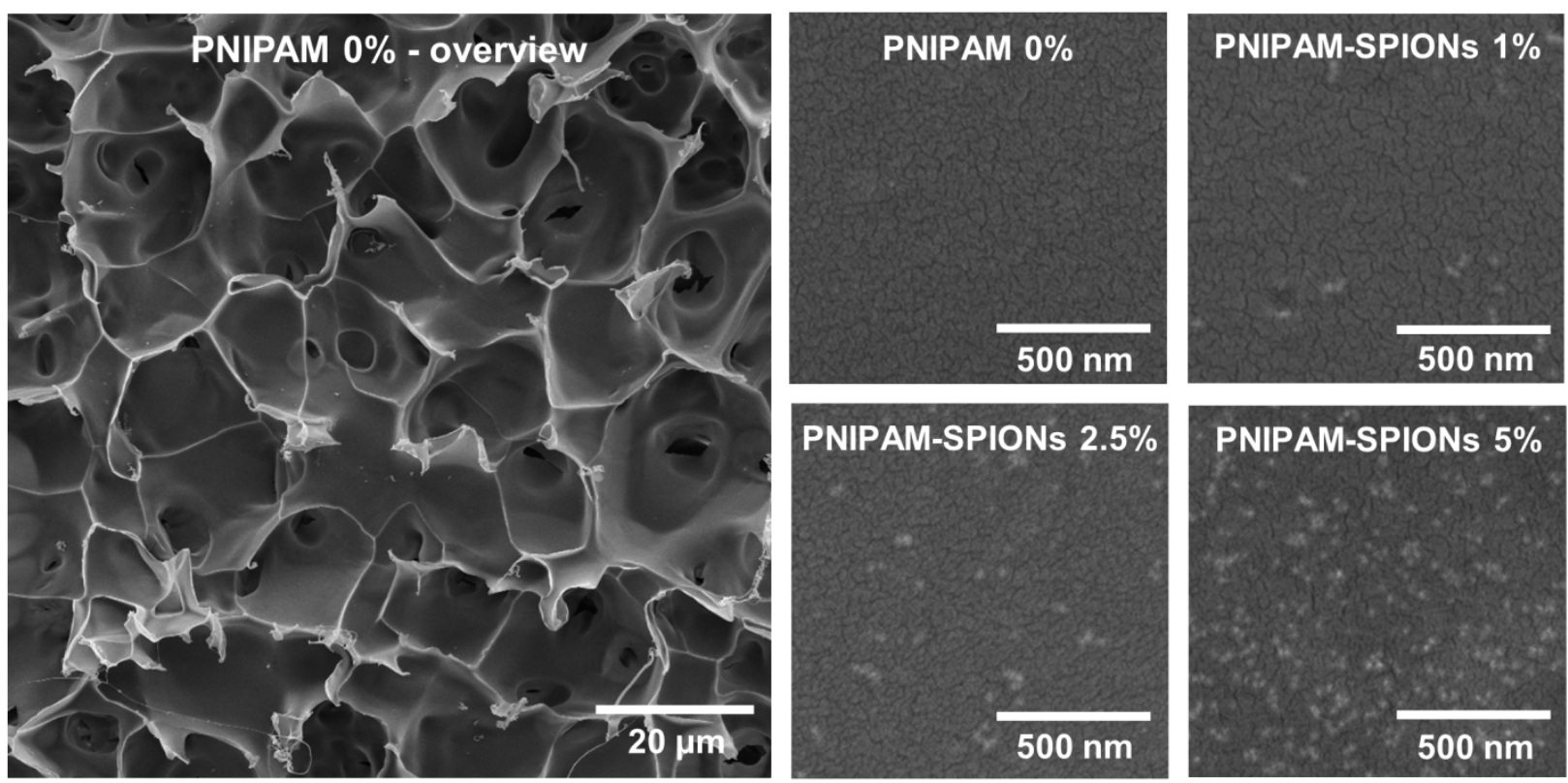

Fig. 2. SEM images of hydrogels loaded with increasing amount of SPIONs. On the left and overview of a PNIPAM hydrogels in its freeze-dried form, on the right high magnification images of the surface of the gels where the nanoparticles can be observed (white dots).

The swelling behavior of the gels was analyzed from 25 to $55^{\circ} \mathrm{C}$ to investigate the effect of the nanoparticles in the hydrogels. Fig. 3A shows the mass swelling ratio values (Q) for all magnetic hydrogels. As the temperature increased the swelling ratio of all systems decreased, and at the lower critical solution temperature (LCST), the temperature above which PNIPAM chains transition to a collapsed insoluble state, they exhibited a sharp de-swelling behavior. Increasing the concentration of particles in the system did not introduce any significant deviation in the temperature sensitivity of the 
hydrogels. Indeed the LCST was found to be around $34{ }^{\circ} \mathrm{C}$ for all the hydrogels, as is expected for pure PNIPAM [46]. Analogous observations were reported for similar systems [14].

Fig. 3B displays magnetization loops (magnetization vs. applied magnetic field) for PNIPAM hydrogels loaded with $0,1,2.5$, and $5 \% \mathrm{w} / \mathrm{w}$ of SPIONs. As expected the PNIPAM 0\% hydrogel was weakly diamagnetic while the other materials showed a pronounced superparamagnetic behavior that was directly related to the presence of the magnetic nanoparticles. Neither remanence nor coercivity were observed, thus confirming SEM morphology analysis indicating that the particles were homogeneously incorporated through the polymer matrix and non-agglomerated. For SPIONs-loaded hydrogels the saturation magnetization ranged between 0.7 and $3.2 \mathrm{emu} / \mathrm{g}$ and was dependent on the iron concentration in the different synthetic formulations. However the dependence was not linear, in particular there was a clear non-linearity between the saturation magnetization of PNIPAM-SPIONs 2.5\% and 5\%, which may be related to difference in final iron content in the hydrogels. PNIPAM-SPIONs 5\% could be more prone to lose SPIONs during the post synthesis washing steps.
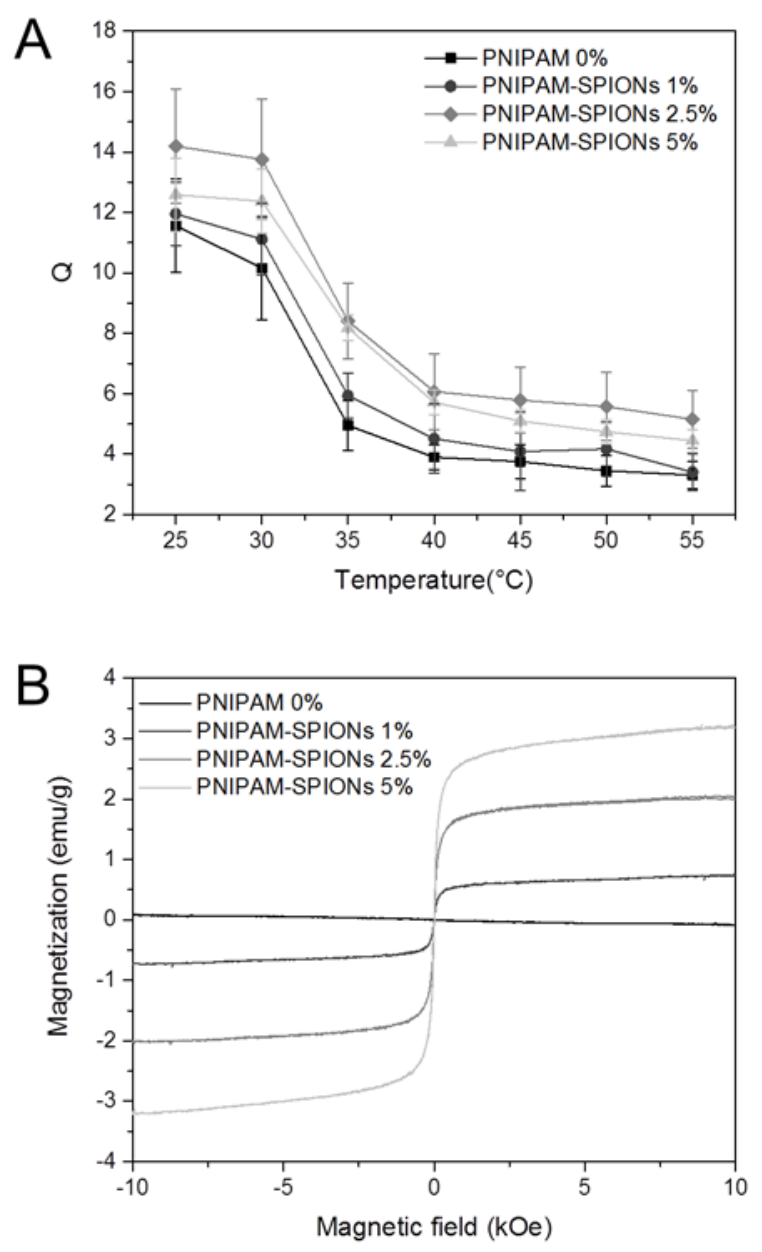
Fig. 3. SPIONs concentration effect on the thermal and magnetic behavior of the hydrogels. Mass swelling ratio (A) from 25 to $55^{\circ} \mathrm{C}$ for the hydrogels loaded with different amount of SPIONs ( $\mathrm{n}=3$, average $\pm \mathrm{SD}$ ). Magnetization $M H$ curves of magnetic hydrogels synthesized with different SPIONs concentrations (B).

\subsection{AMF heating and dynamic phase change}

Heating of magnetic hydrogels can be achieved by exciting the SPIONs in the polymeric matrix via AMF [14], [19]. Moreover thermo-responsive polymers loaded with superparamagnetic nanoparticles can change phase upon magnetic stimulation and can be used for drug release[47]-[49]. In this work we developed magnetic hydrogel substrates and then performed AMF heating measures at room temperature to assess the dependence of magnetic field intensity, frequency and iron concentration on the hydrogel temperature increase. As reported in Fig. 4A the maximum temperature rise was obtained for the PNIPAM-SPIONs 5\% excited with a strong AMF ( $\mathrm{f}=523 \mathrm{kHz}, \mathrm{H}=15.9 \mathrm{kA} / \mathrm{m}$ ). PNIPAM-SPIONs 2.5\% still produced a detectable increase in temperature, while the PNIPAM-SPIONs $1 \%$ hydrogel had a heating capacity similar to that of pure PNIPAM. The use of a mild AMF ( $\mathrm{f}=110 \mathrm{kHz}, \mathrm{H}=19.9 \mathrm{kA} / \mathrm{m}$ ) resulted in a weak increase in temperature for all the hydrogel formulations. As a consequence the study in this condition was not pursued, even though for the given combination of field and frequency the criterion for a maximal exposure were observed $\left(\mathrm{H} \cdot \mathrm{f} \leq 5 \times 10^{9} \mathrm{~A} / \mathrm{ms}\right)[50]$.

For phase change experiments we selected the magnetic hydrogel with the highest nanoparticles concentration and chose the stronger AMF parameters (PNIPAM-SPIONs 5\% and $\mathrm{f}=523 \mathrm{kHz}, \mathrm{H}=15.9$ $\mathrm{kA} / \mathrm{m}$ ). In order to increase and stabilize the initial temperature of the hydrogel, a temperature-controlled sample holder was developed. The system, a 3D printed double wall chamber that could be accommodated in the middle of the coil (geometry in Supporting Information), was connected to a thermostatic bath. With this setup it was possible to directly stabilize the initial temperature at $33{ }^{\circ} \mathrm{C}$ for the entire duration of the test (Fig. 4C blue chart representing PNIPAM-SPIONs 5\% without AMF). Upon cyclic AMF stimulation a reversible phase change of the PNIPAM-SPIONs 5\% hydrogel was observed (Fig. 4C). In our experiments the phase transition started at $34{ }^{\circ} \mathrm{C}$, was completed at $35{ }^{\circ} \mathrm{C}$ and never exceeded $36{ }^{\circ} \mathrm{C}$ ( $n=4$, data in Supporting Information). Moreover when the field was off the temperature quickly dropped (c.a. 5 minutes) and the original swollen phase was immediately recovered. Photographs of hydrogels over the course of cyclic AMF heating show the unheated magnetic hydrogels as a dark opaque mass, and upon cyclic AMF stimulation there was a transition to a translucent gel. This translucent shift was reversible, and indicative of the magnetic hydrogel's temperature-sensitive (and stimuli-induced) behavior to which is possible to associate changes in surface and bulk mechanical properties [30], [35].

Low temperature variations ( $\Delta$ Temperature at $2-3{ }^{\circ} \mathrm{C}$ ) were undesirable or avoided for other studies oriented towards hyperthermia treatment, which were therefore interested in higher temperature variations [19], [49]. However this phenomenon was an interesting result for us since we aim to affect a 
mechanical change to a cell growth substrate with minimal perturbation of the surrounding environment. Therefore an increase in the hydrogel temperature of only $2-3{ }^{\circ} \mathrm{C}$ that resulted in magnetically-triggered de-swelling/swelling cycles, and that does not adversely affect cell behavior per se, matches our objective. Moreover the hydrogel temperature during magnetic excitation was found to be stable around $35{ }^{\circ} \mathrm{C}$ well below the physiological limit for cells (around $42{ }^{\circ} \mathrm{C}$ ) [51], [52]. While conventional cell culture is conducted at $37{ }^{\circ} \mathrm{C}$, there have been reports of cell culture carried out at lower temperatures with no adverse effects on cell viability [20], [53]. Therefore, this magneto thermo-responsive hydrogel offers an interesting and unique means to study cell behavior on a dynamic substrate. Nevertheless the system can be further improved using, for example, co-polymers [54] where the LCST can be tuned around $37^{\circ} \mathrm{C}$.
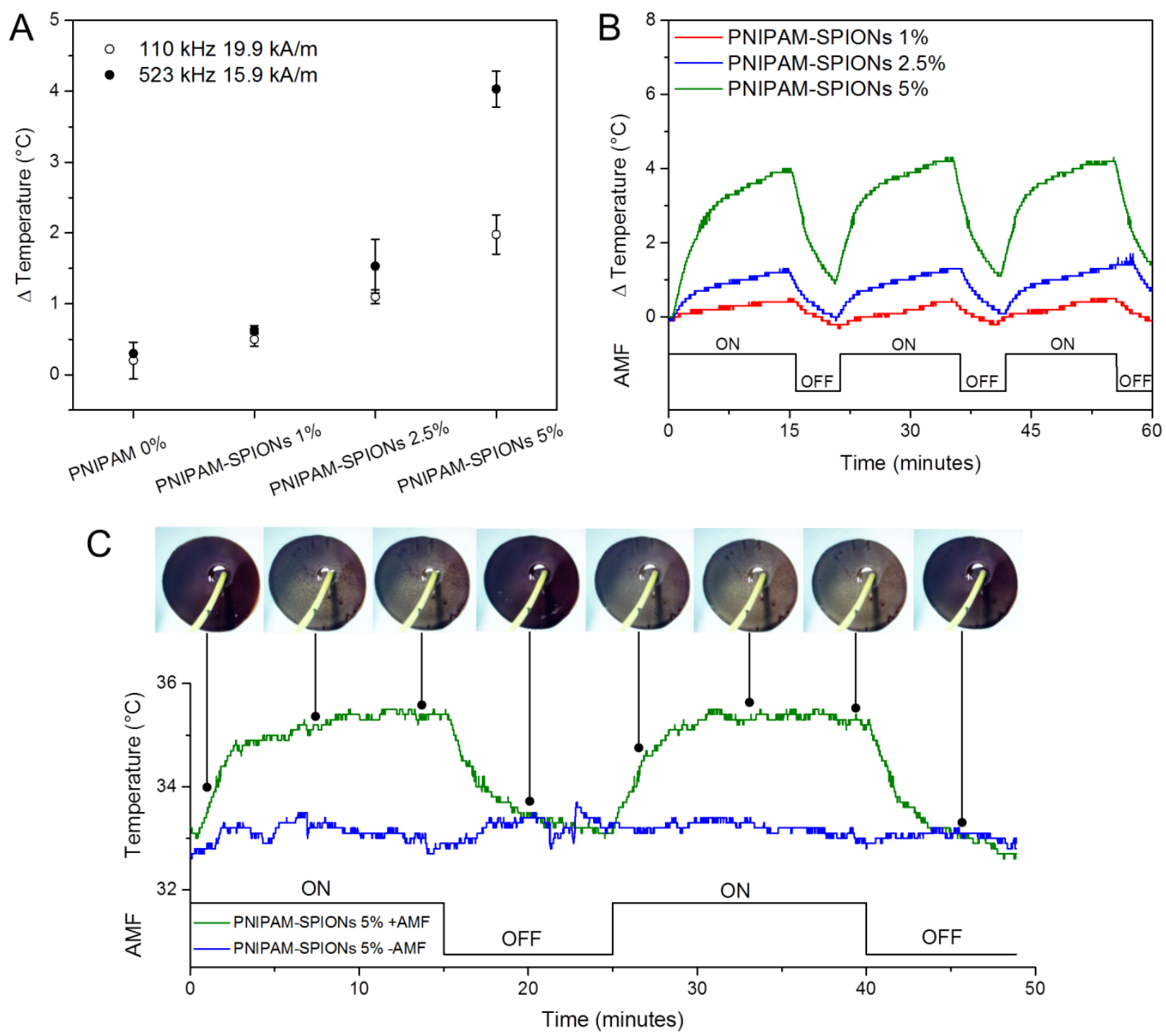

Fig. 4. Incremental temperature increase as a function of iron concentration, and magnetic field strength and frequency (A). The values were recorded for equilibrated hydrogel in Milli-Q water after exciting them with the AMF for 15 minutes ( $n=3, \pm S D$ ). Reversible, iron concentration-dependent heating profiles of magnetic hydrogels stimulated with 3 cycles of AMF (523 kHz, $15.9 \mathrm{kA} / \mathrm{m}$ ) (B). Reversible magnetic field-dependent temperature 
profile of the magnetic hydrogels (PNIPAM-SPIONs 5\% +AMF) upon cyclic exposure to AMF (523 kHz, 15.9 $\mathrm{kA} / \mathrm{m}$ ) or without the magnetic excitation (PNIPAM-SPIONs 5\% -AMF) (C). Pictures of the hydrogels during the magnetic cycles (taken every 5 minutes) were reported to show how the AMF stimulation determined the collapse of the hydrogel that quickly re-acquired its initial conformation when the magnetic field was off.

\subsection{Biocompatibility of magnetic hydrogels}

The biocompatibility of magnetic hydrogel extracts was tested on NIH 3T3 murine fibroblast cells. Fibroblasts, and in particular this standardized cell line, have been already investigated on stimuli responsive substrates [26], [55], [56] and were therefore chosen for the viability tests. Following ISO 10993-5 guidelines, magnetic hydrogels and controls were incubated at $37^{\circ} \mathrm{C}$ overnight in complete cell culture medium (Dulbecco's Modified Eagle’s Medium supplemented with 10\% v/v calf bovine serum, 2 $\mathrm{mM}$ L-glutamine 200mM, and 1\% v/v Penicillin-Streptomycin, cDMEM) and extracts of the solution were tested on fibroblasts for adverse effects. In order to test whether AMF-induced swelling/de-swelling resulted in leaching of potentially cytotoxic byproducts, magnetic hydrogels were incubated in cDMEM at $25{ }^{\circ} \mathrm{C}$ overnight and then subjected to three swelling and de-swelling cycles (15 min at $37{ }^{\circ} \mathrm{C}$ and 5 min at $25^{\circ} \mathrm{C}$ ). Cell viability was measured via two methods: i) quantifying lactate dehydrogenase (LDH), an intracellular enzyme release upon damage to the cellular membrane, in the supernatant of cells exposed to magnetic hydrogel extracts; and ii) metabolic activity was quantified by measuring the active cellular reduction of non-fluorescent resazurin to the fluorescent molecule resorufin by cells exposed to magnetic hydrogel extracts [38], [57]. 3T3 cells were exposed to $0.2 \% \mathrm{v} / \mathrm{v}$ Triton X-100, a nonionic surfactant which ruptures the cellular membrane, as a positive control. The results of the LDH assay (Fig. 5A) and the resazurin assay (Fig. 5B) showed no significant toxicity for any of the extract solutions in comparison to the control treated with cDMEM (media) only. These data show that there are no toxic leachables that are released by the magnetic hydrogels, either when incubated at $37^{\circ} \mathrm{C}$ or when subjected to repeated swelling and de-swelling cycles. Moreover, the constituent hydrogel monomers were tested in a dosedependent manner for toxicity (Supplementary Information), and there was no observable effect from the initial monomers. While further studies are necessary to validate the feasibility of the magnetic hydrogels as a dynamic cell culture material, these initial findings indicate that they are biologically non-toxic substrate for cell growth [58]. 

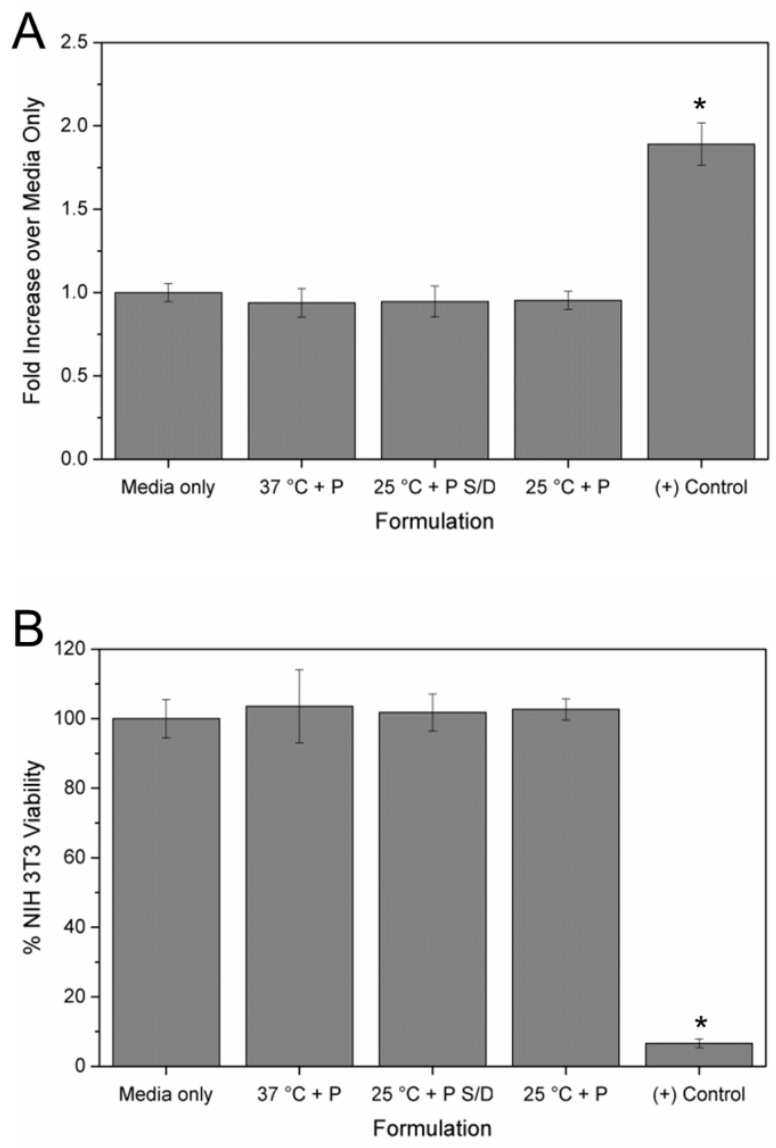

Fig. 5. Cytotoxicity of magnetic hydrogel extracts exposed to NIH 3T3 murine fibroblasts measured by LDH release due to membrane damage (A), and mitochondrial activity via the reduction of resazurin to resorufin (B). Positive control for cytotoxicity was monitored by exposure to $0.2 \% \mathrm{v} / \mathrm{v}$ Triton X-100. Magnetic hydrogels were either incubated in Dulbecco's Modified Eagle's Medium supplemented with $10 \% \mathrm{v} / \mathrm{v}$ calf bovine serum, $2 \mathrm{mM}$ Lglutamine, and $1 \% \mathrm{v} / \mathrm{v}$ penicillin-streptomycin (CDMEM) overnight at $37^{\circ} \mathrm{C}\left(37^{\circ} \mathrm{C}+\mathrm{P}\right)$, in cDMEM at $25^{\circ} \mathrm{C}$ with swelling and de-swelling cycles $\left(25^{\circ} \mathrm{C}+\mathrm{P}\right.$ S/D), or in cDMEM at $25^{\circ} \mathrm{C}\left(25^{\circ} \mathrm{C}+\mathrm{P}\right)$. Tests were done in triplicate with three replicates per test. All measurements were compared to the mean of the negative control (media only), and comparison of means was conducted with a one-way ANOVA and Tukey's HSD test ( $p$-value $=0.05)$. * Indicates a statistically significant difference from the negative control.

\section{Conclusion}

We have presented the fabrication and characterization of a magnetic thermo-responsive hydrogel based on poly-N-isopropylacrylamide embedded with superparamagnetic iron oxide nanoparticles. $22 \mathrm{~nm}$ SPIONs, synthesized by thermal decomposition, were first transferred to water and then incorporated in the hydrogel at different ratios of 1, 2.5 and 5\% w/w. Nanoparticles were evenly dispersed in the hydrogels and displayed thermo-responsive and superparamagnetic behavior. The application of an AMF was utilized to successfully trigger phase changes $\left(33-35{ }^{\circ} \mathrm{C}\right)$ of the hydrogel loaded with $5 \%$ w/w of 
particles. In particular the mechanical properties of the substrate can be varied by AMF in a narrow temperature range, minimizing the perturbation caused by drastic and broad changes in temperature on the surrounding environment. That is, there are significant physiological ramifications for large temperature changes with a living system and by tuning the physical response of the hydrogel within a narrow temperature range $\left(2-3^{\circ} \mathrm{C}\right)$ it is possible to alter the hydrogel's physical properties without negatively affecting the surrounding biological milieu. Moreover, indirect cytotoxicity assays showed the biocompatibility of these materials. Considering the obtained results, these magnetic thermo-responsive hydrogels combined desired properties for mechanobiology applications and may be considered promising candidates as dynamic cellular substrates.

\section{Acknowledgment}

This work was supported by the National Center of Competence in Research Bio-Inspired Materials, the Adolphe Merkle Foundation and the University of Fribourg. The authors kindly acknowledge David Bossert for the purification of the monomers, Ana Milosevic for the first biocompatibility tests and Dr. Balog for the support with DLS.

\section{References}

[1] I. Levental, P. C. Georges, and P. A. Janmey, "Soft biological materials and their impact on cell function,” Soft Matter, vol. 2, pp. 1-9, 2007.

[2] A. S. Hoffman, "Hydrogels for biomedical applications," Adv. Drug Deliv. Rev., vol. 64, no. SUPPL., pp. 18-23, 2012.

[3] K. Y. Lee and D. J. Mooney, "Hydrogels for tissue engineering,” Chem. Rev., vol. 101, no. 7, pp. 1869-1879, 2001.

[4] J. L. Drury and D. J. Mooney, "Hydrogels for tissue engineering: Scaffold design variables and applications,” Biomaterials, vol. 24, no. 24, pp. 4337-4351, 2003.

[5] Y. Li, G. Huang, X. Zhang, B. Li, Y. Chen, T. Lu, T. J. Lu, and F. Xu, "Magnetic hydrogels and their potential biomedical applications,” Adv. Funct. Mater., vol. 23, no. 6, pp. 660-672, 2013.

[6] R. Fuhrer, E. K. Athanassiou, N. A. Luechinger, and W. J. Stark, “Crosslinking metal nanoparticles into the polymer backbone of hydrogels enables preparation of soft, magnetic fielddriven actuators with muscle-like flexibility,” Small, vol. 5, no. 3, pp. 383-388, 2009.

[7] A. Tampieri, E. Landi, F. Valentini, M. Sandri, T. D’Alessandro, V. Dediu, and M. Marcacci, “A conceptually new type of bio-hybrid scaffold for bone regeneration.,” Nanotechnology, vol. 22, no. 1, p. 015104, 2011. 
[8] C. S. Brazel, "Magnetothermally-responsive nanomaterials: Combining magnetic nanostructures and thermally-sensitive polymers for triggered drug release,” Pharm. Res., vol. 26, no. 3, pp. 644656, 2009.

[9] T. Y. Liu, S. H. Hu, D. M. Liu, S. Y. Chen, and I. W. Chen, "Biomedical nanoparticle carriers with combined thermal and magnetic responses,” Nano Today, vol. 4, no. 1, pp. 52-65, 2009.

[10] B. Mehdaoui, A. Meffre, J. Carrey, S. Lachaize, L. M. Lacroix, M. Gougeon, B. Chaudret, and M. Respaud, "Optimal size of nanoparticles for magnetic hyperthermia: A combined theoretical and experimental study,” Adv. Funct. Mater., vol. 21, no. 23, pp. 4573-4581, 2011.

[11] R. E. E. Rosensweig, "Heating magnetic fluid with alternating magnetic field," J. Magn. Magn. Mater., vol. 252, no. 0, pp. 370-374, 2002.

[12] R. Hergt, S. Dutz, R. Müller, and M. Zeisberger, "Magnetic particle hyperthermia: nanoparticle magnetism and materials development for cancer therapy,” J. Phys. Condens. Matter, vol. 18, no. 38, pp. S2919-S2934, 2006.

[13] M. A. Ward and T. K. Georgiou, "Thermoresponsive polymers for biomedical applications," Polymers (Basel)., vol. 3, no. 3, pp. 1215-1242, 2011.

[14] N. S. Satarkar and J. Zach Hilt, "Hydrogel nanocomposites as remote-controlled biomaterials," Acta Biomater., vol. 4, no. 1, pp. 11-16, 2008.

[15] J. E. Wong, A. K. Gaharwar, D. Müller-Schulte, D. Bahadur, and W. Richtering, "Dual-stimuli responsive PNiPAM microgel achieved via layer-by-layer assembly: Magnetic and thermoresponsive,” J. Colloid Interface Sci., vol. 324, no. 1-2, pp. 47-54, 2008.

[16] R. Regmi, S. R. Bhattarai, C. Sudakar, A. S. Wani, R. Cunningham, P. P. Vaishnava, R. Naik, D. Oupicky, and G. Lawes, "Hyperthermia controlled rapid drug release from thermosensitive magnetic microgels,” J. Mater. Chem., vol. 20, no. 29, pp. 6158-6163, 2010.

[17] S. B. Campbell, M. Patenaude, and T. Hoare, "Injectable superparamagnets: Highly elastic and degradable poly(N-isopropylacrylamide)-superparamagnetic iron oxide nanoparticle (SPION) composite hydrogels,” Biomacromolecules, vol. 14, no. 3, pp. 644-653, 2013.

[18] A. T. Paulino, A. G. B. Pereira, A. R. Fajardo, K. Erickson, M. J. Kipper, E. C. Muniz, L. A. Belfiore, and E. B. Tambourgi, "Natural polymer-based magnetic hydrogels: Potential vectors for remote-controlled drug release,” Carbohydr. Polym., vol. 90, no. 3, pp. 1216-1225, 2012.

[19] K. L. Ang, S. Venkatraman, and R. V. Ramanujan, "Magnetic PNIPA hydrogels for hyperthermia applications in cancer therapy,” Mater. Sci. Eng. C, vol. 27, no. 3, pp. 347-351, 2007.

[20] K. A. Davis, K. A. Burke, P. T. Mather, and J. H. Henderson, "Dynamic cell behavior on shape memory polymer substrates,” Biomaterials, vol. 32, no. 9, pp. 2285-2293, 2011.

[21] H. Y. Yoshikawa, F. F. Rossetti, S. Kaufmann, T. Kaindl, J. Madsen, U. Engel, A. L. Lewis, S. P. Armes, and M. Tanaka, "Quantitative Evaluation of Mechanoresponse of Cells on Dynamically Tunable Hydrogels,” J. Am. Chem. Soc., no. 133, pp. 1367-1374, 2011.

[22] M. Guvendiren and J. a Burdick, "Stiffening hydrogels to probe short- and long-term cellular responses to dynamic mechanics.,” Nat. Commun., vol. 3, p. 792, 2012.

[23] T. Velnar, T. Bailey, and V. Smrkolj, "The wound healing process: an overview of the cellular and 
molecular mechanisms.,” J. Int. Med. Res., vol. 37, no. 5, pp. 1528-42, 2009.

[24] S. Huang and D. E. Ingber, “Cell tension, matrix mechanics, and cancer development," Cancer Cell, vol. 8, no. 3, pp. 175-176, 2005.

[25] K. Uto, M. Ebara, and T. Aoyagi, “Temperature-responsive poly( $\varepsilon$-caprolactone) cell culture platform with dynamically tunable nano-roughness and elasticity for control of myoblast morphology,” Int. J. Mol. Sci., vol. 15, no. 1, pp. 1511-1524, 2014.

[26] K. Yamaki, I. Harada, M. Goto, C. S. Cho, and T. Akaike, "Regulation of cellular morphology using temperature-responsive hydrogel for integrin-mediated mechanical force stimulation,” Biomaterials, vol. 30, no. 7, pp. 1421-1427, 2009.

[27] J. D. Kiang, J. H. Wen, J. C. Del ??lamo, and A. J. Engler, "Dynamic and reversible surface topography influences cell morphology,” J. Biomed. Mater. Res. - Part A, vol. 101 A, no. 8, pp. 2313-2321, 2013.

[28] W. Li, T. Gong, H. Chen, L. Wang, J. Li, and S. Zhou, “Tuning surface micropattern features using a shape memory functional polymer,” RSC Adv., vol. 3, no. 25, p. 9865, 2013.

[29] M. Mayer, R. Rabindranath, J. Börner, E. Hörner, A. Bentz, J. Salgado, H. Han, H. Böse, J. Probst, M. Shamonin, G. J. Monkman, and G. Schlunck, "Ultra-Soft PDMS-Based Magnetoactive Elastomers as Dynamic Cell Culture Substrata,” PLoS One, vol. 8, no. 10, 2013.

[30] X. Sui, Q. Chen, M. A. Hempenius, and G. J. Vancso, "Probing the collapse dynamics of poly(Nisopropylacrylamide) brushes by AFM: Effects of Co-nonsolvency and grafting densities,” Small, vol. 7, no. 10, pp. 1440-1447, 2011.

[31] J. Park, K. An, Y. Hwang, J.-G. Park, H.-J. Noh, J.-Y. Kim, J.-H. Park, N.-M. Hwang, and T. Hyeon, "Ultra-large-scale syntheses of monodisperse nanocrystals,” Nat. Mater., vol. 3, no. 12, pp. 891-895, 2004.

[32] M. Lattuada and T. A. Hatton, "Functionalization of monodisperse magnetic nanoparticles," Langmuir, vol. 23, no. 4, pp. 2158-2168, 2007.

[33] S. Boutry, D. Forge, C. Burtea, I. Mahieu, O. Murariu, S. Laurent, L. Vander Elst, and R. N. Muller, "How to quantify iron in an aqueous or biological matrix: A technical note," Contrast Media Mol. Imaging, vol. 4, no. 6, pp. 299-304, 2009.

[34] T. Vignaud, H. Ennomani, and M. Théry, "Polyacrylamide Hydrogel Micropatterning,” Methods Cell Biol., vol. 120, pp. 93-116, 2014.

[35] Z. Li, J. Shen, H. Ma, X. Lu, M. Shi, N. Li, and M. Ye, "Preparation and characterization of sodium alginate/poly(N- isopropylacrylamide)/clay semi-IPN magnetic hydrogels,” Polym. Bull., vol. 68, no. 4, pp. 1153-1169, 2012.

[36] M. Kallumadil, M. Tada, T. Nakagawa, M. Abe, P. Southern, and Q. A. Pankhurst, "Journal of Magnetism and Magnetic Materials Suitability of commercial colloids for magnetic hyperthermia," J. Magn. Magn. Mater., vol. 321, pp. 1509-1513, 2009.

[37] “International Standard - biological evaluation of medical devices - Part 5.”

[38] J. O’Brien, I. Wilson, T. Orton, and F. Pognan, "Investigation of the Alamar Blue (resazurin) fluorescent dye for the assessment of mammalian cell cytotoxicity,” Eur. J. Biochem., vol. 267, no. 
17, pp. 5421-5426, 2000.

[39] Y. Dieckmann, H. Co, H. Hofmann, and A. Petri-fink, "Particle Size Distribution Measurements of Manganese-Doped ZnS Nanoparticles,” Anal. Chem., vol. 81, no. 10, pp. 3889-3895, 2013.

[40] S. Wagner, J. Schnorr, H. Pilgrimm, B. Hamm, and M. Taupitz, "Monomer-Coated Very Small Superparamagnetic Iron Oxide,” Invest. Radiol., vol. 37, no. 4, pp. 167-177, 2002.

[41] I. Ojea-Jiménez, F. M. Romero, N. G. Bastu, and V. Puntes, "Small Gold Nanoparticles Synthesized with Sodium Citrate and Heavy Water : Insights into the Reaction Mechanism," pp. 1800-1804, 2010.

[42] J. Park and J. S. Shumaker-Parry, "Structural Study of Citrate Layers on Gold Nanoparticles: Role of Intermolecular Interactions in Stabilizing Nanoparticles,” 2014.

[43] J. S. Salazar, L. Perez, O. De Abril, L. T. Phuoc, D. Ihiawakrim, M. Vazquez, J. Greneche, S. Begin-colin, and G. Pourroy, "Magnetic Iron Oxide Nanoparticles in 10 - 40 nm Range :

Composition in Terms of Magnetite / Maghemite Ratio and Effect on the Magnetic Properties,” Chem. Mater., pp. 1379-1386, 2011.

[44] C. Lin, R. Chiang, J. Wang, T. Sung, and C. Lin, "Magnetic properties of monodisperse iron oxide nanoparticles Magnetic properties of monodisperse iron oxide nanoparticles,” J. Appl. Phys., vol. 710, pp. 97-100, 2006.

[45] A. Lak, M. Kraken, F. Ludwig, A. Kornowski, D. Eberbeck, S. Sievers, and F. J. Litterst, "Size dependent structural and magnetic properties of,” Nanoscale, vol. 5, pp. 12286-12295, 2013.

[46] S. Hirotsu, Y. Hirokawa, and T. Tanaka, "Volume-phase transitions of ionized Nisopropylacrylamide gels,” J. Chem. Phys., vol. 87, no. 2, pp. 1392-1395, 1987.

[47] N. S. Satarkar, W. Zhang, R. E. Eitel, and J. Z. Hilt, "Magnetic hydrogel nanocomposites as remote controlled microfluidic valves.,” Lab Chip, vol. 9, no. 12, pp. 1773-1779, 2009.

[48] X. Wang, S. Yang, C. Wang, L. Chen, and S. Chen, "Multifunctional Hydrogels with Temperature , Ion , and Magnetocaloric Stimuli-Responsive Performances,” pp. 759-768.

[49] S. A. Meenach, K. W. Anderson, and J. Z. Hilt, "Synthesis and Characterization of Thermoresponsive Poly(ethylene glycol)-Based Hydrogels and Their Magnetic Nanocomposites," J. Polym. Sci. Part A Polym. Chem., vol. 48, no. 1, pp. 3229-3235, 2010.

[50] R. Hergt, S. Dutz, R. Michael, and M. Röder, "Effects of size distribution on hysteresis losses of magnetic nanoparticles for hyperthermia.,” J. Phys. Condens. Matter, vol. 20, no. 38, p. 385214, 2008 .

[51] J. Overgaard, "Effect of hyperthermia on malignant cells in vivo. A review and a hypothesis.," Cancer, vol. 39, no. 6, pp. 2637-46, 1977.

[52] R. I. Morimoto, “Cells in Stress: Transcriptional Activation of Heat Shock Genes.” Science.

[53] T. Gong, K. Zhao, G. Yang, J. Li, H. Chen, Y. Chen, and S. Zhou, "The Control of Mesenchymal Stem Cell Differentiation Using Dynamically Tunable Surface Microgrooves,” Adv. Healthc. Mater., vol. 3, no. 10, pp. 1608-1619, 2014.

[54] J. M. Rathfon and G. N. Tew, "Synthesis of thermoresponsive poly(N-isopropylmethacrylamide) 
and poly(acrylic acid) block copolymers via post-functionalization of poly(N-

methacryloxysuccinimide),” Polymer (Guildf)., vol. 49, no. 7, pp. 1761-1769, 2008.

[55] M. Ebara, K. Uto, N. Idota, J. M. Hoffman, and T. Aoyagi, "Shape-memory surface with dynamically tunable nano-geometry activated by body heat,” Adv. Mater., vol. 24, no. 2, pp. 273278, 2012.

[56] M. T. Frey and Y. Wang, "A photo-modulatable material for probing cellular responses to substrate rigidity,” Soft Matter, vol. 5, no. 9, pp. 1918-1924, 2009.

[57] G. Fotakis and J. A. Timbrell, "In vitro cytotoxicity assays: Comparison of LDH, neutral red, MTT and protein assay in hepatoma cell lines following exposure to cadmium chloride,” Toxicol. Lett., vol. 160, no. 2, pp. 171-177, 2006.

[58] S. A. Meenach, A. A. Anderson, M. Suthar, K. W. Anderson, and J. Z. Hilt, "Biocompatibility analysis of magnetic hydrogel nanocomposites based on poly(N-isopropylacrylamide) and iron oxide,” J. Biomed. Mater. Res. - Part A, vol. 91, no. 3, pp. 903-909, 2009. 


\section{Supporting Information}

\section{Dynamic and biocompatible thermo-responsive magnetic hydrogels that respond to an alternating magnetic field.}

Federica Crippa $^{\mathrm{a}}$, Thomas L. Moore ${ }^{\mathrm{a}}$, Mariangela Mortato ${ }^{\mathrm{a}}$, Christoph Geers ${ }^{\mathrm{a}}$, Laetitia Haeni ${ }^{\mathrm{a}}$, Ann M. Hirt $^{\mathrm{b}}$, Barbara Rothen-Rutishauser ${ }^{\mathrm{a}}$, Alke Petri-Fink ${ }^{\mathrm{a}, \mathrm{c}, *}$

${ }^{a}$ Adolphe Merkle Institute, University of Fribourg, Chemin des Verdiers 4, CH-1700 Fribourg, Switzerland

${ }^{b}$ Institute for Geophysics, ETH Zurich, Sonneggstrasse 5, CH-8092 Zurich, Switzerland

${ }^{c}$ Chemistry Department, University of Fribourg, Chemin du Musée 9, CH-1700 Fribourg Switzerland

* Corresponding author: Chemistry Department, University of Fribourg, Chemin du Musée 9, CH-1700 Fribourg, Switzerland. E-mail: alke.fink@unifr.ch (A. Petri-Fink).

\section{A.1 SPIONs synthesis}

\section{A.1.1 Iron oleate-complex synthesis}

$8.66 \mathrm{~g}$ of iron (III) chloride hexahydrate and $29.792 \mathrm{~g}$ of sodium oleate were dissolved in a mixture of 64 $\mathrm{ml}$ ethanol, $48 \mathrm{ml}$ distilled water and $112 \mathrm{ml}$ hexane. The reaction mixture was heated at $70^{\circ} \mathrm{C}$ and kept at reflux for four hours under magnetic stirring. Afterwards the organic phase was washed 3 times with 50 $\mathrm{ml}$ distilled water in a separatory funnel, the hexane was evaporated and the final iron oleate-complex was stored in glass flasks at $4^{\circ} \mathrm{C}$.

\section{A.1.2 Synthesis of iron oxide nanoparticles}

Oleic acid SPIONs were synthetized as follow: $14.51 \mathrm{~g}$ of freshly prepared iron oleate-complex and 2.31 $\mathrm{ml}$ of oleic acid (pur. 90\%, Aldrich) were dissolved in $105.3 \mathrm{ml}$ of trioctylamine (pur. $>97 \%$, bpt:367 ${ }^{\circ} \mathrm{C}$, TCI) and degassed at $50^{\circ} \mathrm{C}$ for 2 hours. Then the solution was heated to $320^{\circ} \mathrm{C}$ under a constant flow of argon with an automatized 2-step heating process (Thermostat T Huber): firstly the temperature was quickly increased to $120^{\circ} \mathrm{C}$ (speed rate $10^{\circ} \mathrm{C} / \mathrm{min}$ ), after the heating rate was decreased to $3^{\circ} \mathrm{C} / \mathrm{min}$ and this speed was kept constant until $320^{\circ} \mathrm{C}$. The reaction was then maintained for 60 minutes at the final temperature to allow the growth of the crystal. After air mediated cooling, hexane and ethanol were added to precipitate the NPs and a multi steps centrifugation was performed to separate the iron oxide nanocrystals. 

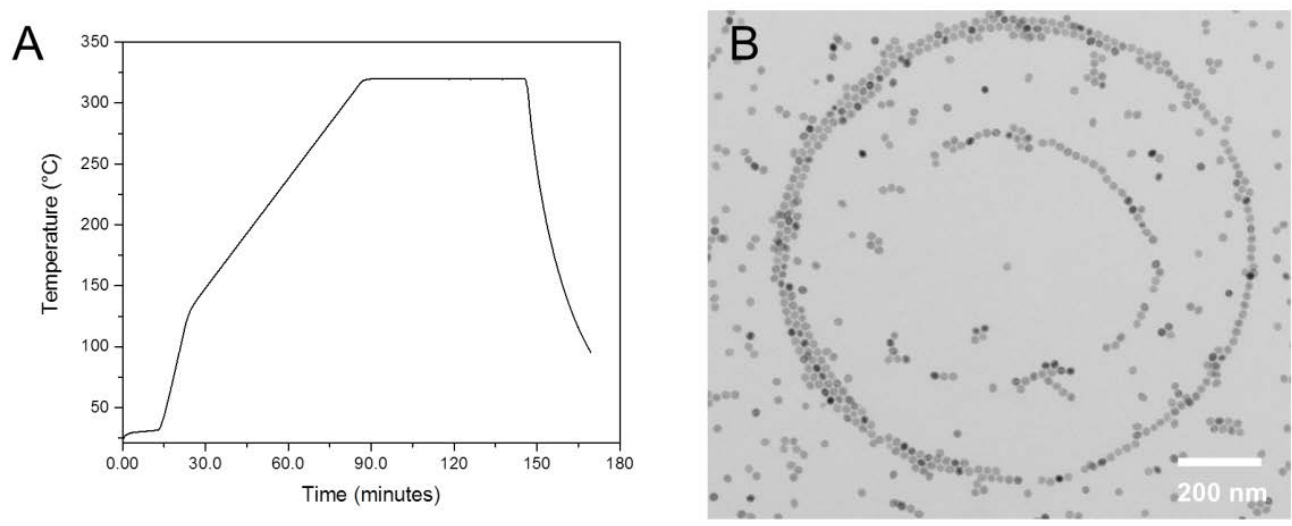

Fig. A.1. Heating ramp used for SPIONs synthesis (A) and TEM image of the oleic acid coated nanocrystals (B).

\section{A.2 AMF heating and dynamic phase change}

\section{A.2.1 Temperature-controlled sample holder}

For phase change experiments a temperature-controlled sample holder was developed. The design render with a CAD software (FreeCAD 0.15) and realized with a 3D printer (Form 2, Formlabs) in hard plastic.
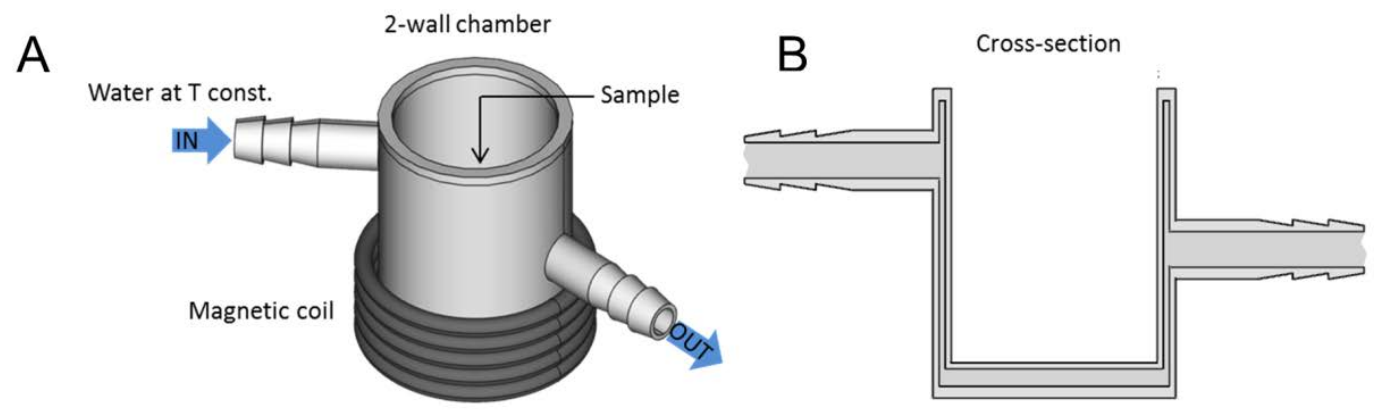

Fig. A.2. Schematic of the temperature-controlled sample holder (A) and its cross-section (B).

\section{A.2.2. Temperature raise during AMF stimulation}

AMF-triggered phase change was obtained for the hydrogel containing 5\% of SPIONs after having stabilized in water at temperatures around $33^{\circ} \mathrm{C}$. In the gels were allowed to equilibrate for 1 hour and then excited with magnetic an AMF on for 15 minutes and off for 10 minutes. For the hydrogels $(n=4)$ the maximum temperature recorded with the fiber optic cables was always below $35.5^{\circ} \mathrm{C}$. 


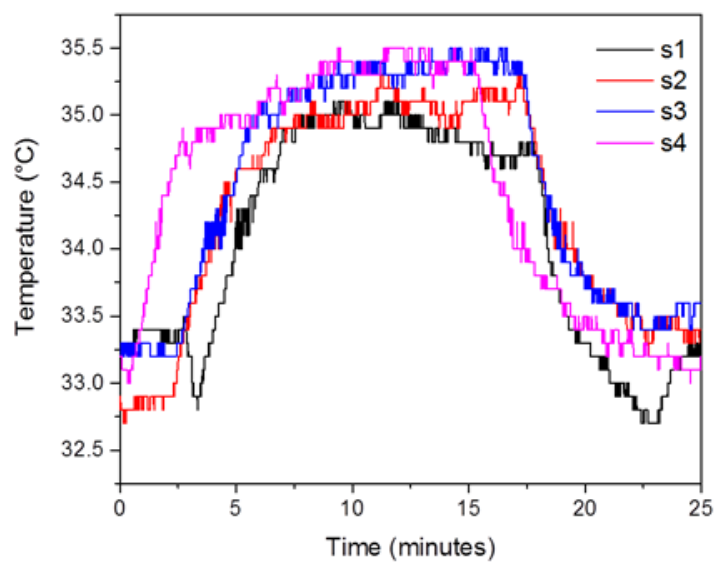

Fig. A.3. Temperature profile of the magnetic hydrogels (PNIPAM-SPIONs $5 \%, n=4$ ) upon exposure to AMF (523 kHz, 15.9 kA/m).

\section{A.3 Viability quantification - control experiments with hydrogel pre-cursor monomers}

As control experiments NIH 3T3 murine fibroblasts were exposed to increasing concentrations of NIPAM and BIS. Specifically cells were exposed to NIPAM solutions at 10, 100, 500, 1000 and $10000 \mu \mathrm{M}$ and BIS solutions at $0.1,1,5,10$ and $100 \mu \mathrm{M}$. Positive control for cytotoxicity was monitored by exposure to $0.2 \mathrm{v} / \mathrm{v} \%$ Triton X-100 while for negative control fresh media was applied on the cells. The obtained data showed that at the given concentrations both monomers are not toxic for the cells.
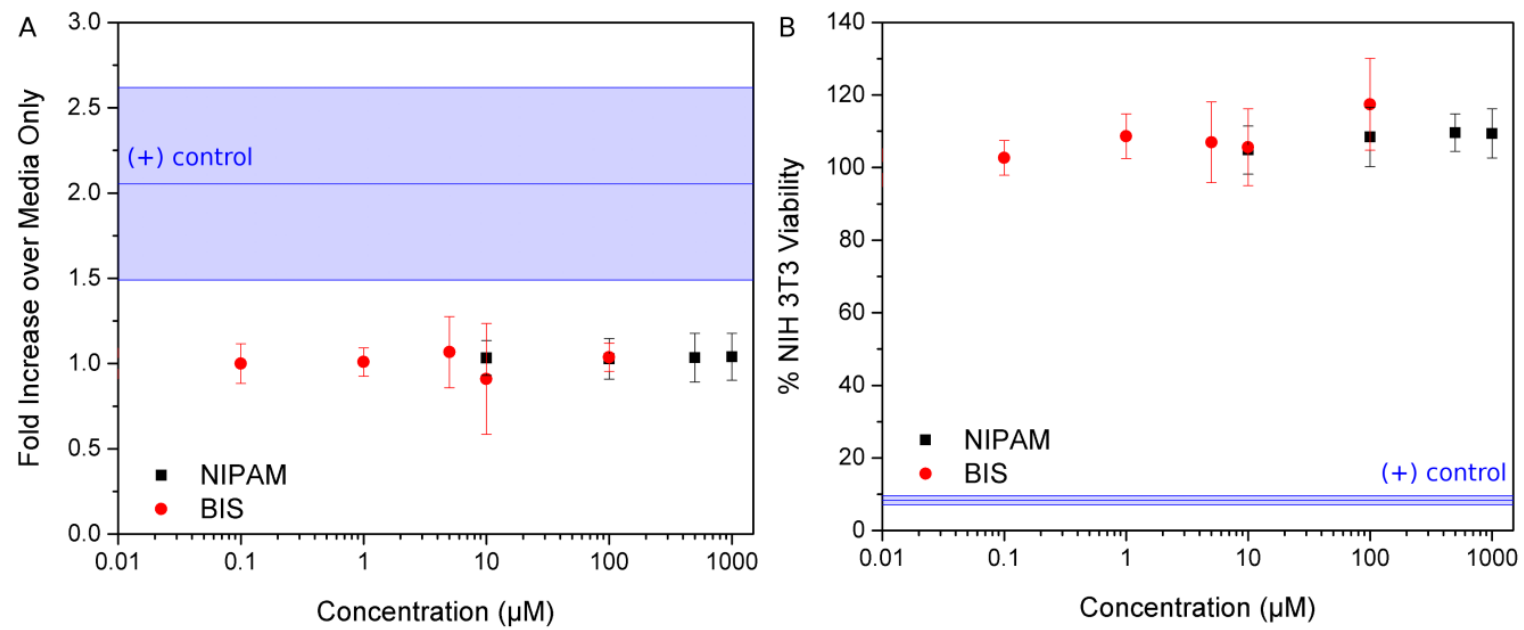

Fig. A.4. Cytotoxicity of NIPAM and BIS monomers exposed to NIH 3T3 murine fibroblasts measured by $\mathrm{LDH}$ release due to membrane damage (A), and mitochondrial activity via the reduction of resazurin to resorufin (B). Tests were done in triplicate with three replicates per test and all the measurements were compared to the mean of the negative control (media only). 